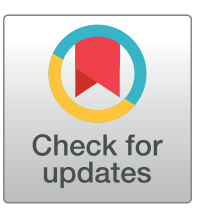

f open access

Citation: Meoni G, Ghini V, Maggi L, Vignoli A, Mazzoni A, Salvati L, et al. (2021) Metabolomic/ lipidomic profiling of COVID-19 and individual response to tocilizumab. PLoS Pathog 17(2): e1009243. https://doi.org/10.1371/journal. ppat.1009243

Editor: Kanta Subbarao, The Peter Doherty Institute and Melbourne University, AUSTRALIA

Received: October 28, 2020

Accepted: December 18, 2020

Published: February 1, 2021

Copyright: ๑ 2021 Meoni et al. This is an open access article distributed under the terms of the Creative Commons Attribution License, which permits unrestricted use, distribution, and reproduction in any medium, provided the original author and source are credited.

Data Availability Statement: Metabolomics data have been deposited to the EMBL-EBI MetaboLights database (DOI: 10.1093/nar/ gkz1019, PMID:31691833) with the identifier MTBLS2336. Prior to release date, they can also be retrieved from www.cerm.unifi.it/static/COVID_ ANN.tgz.

Funding: AV and VG are supported by an AIRC fellowship for Italy. This study was supported by funds from the Department of Experimental and Clinical Medicine of University of Florence,
RESEARCH ARTICLE

\section{Metabolomic/lipidomic profiling of COVID-19 and individual response to tocilizumab}

\author{
Gaia Meoni $\oplus^{1,2 \oplus}$, Veronica Ghini ${ }^{1,3 \oplus}$, Laura Maggi $\oplus^{4}$, Alessia Vignoli $\oplus^{1,3}$, \\ Alessio Mazzoni $\mathbb{D}^{4}$, Lorenzo Salvati $\mathbb{C}^{4}$, Manuela Capone $\mathbb{D}^{4}$, Anna Vanni $\mathbb{D}^{4}$, \\ Leonardo Tenori ${ }^{1,5}$, Paolo Fontanari ${ }^{6}$, Federico Lavorini ${ }^{4,7}$, Adriano Peris ${ }^{8}$, \\ Alessandro Bartoloni $\oplus^{4,9}$, Francesco Liotta ${ }^{4,10}$, Lorenzo Cosmi $\oplus^{4,10}$, Claudio Luchinat ${ }^{1,5}$, \\ Francesco Annunziato ${ }^{4,11} *$, Paola Turano ${ }^{1,5 *}$
}

1 Magnetic Resonance Center (CERM), University of Florence, Sesto Fiorentino, Florence, Italy, 2 Giotto Biotech srl, Sesto Fiorentino, Florence, Italy, 3 Consorzio Interuniversitario Risonanze Magnetiche di Metallo Proteine (CIRMMP), University of Florence, Sesto Fiorentino, Florence, Italy, 4 Department of Clinical and Experimental Medicine, University of Florence, Florence, Italy, 5 Department of Chemistry "Ugo Schiff", University of Florence, Sesto Fiorentino, Florence, Italy, 6 Cardiac Anaesthesia and Intensive Care Unit, Careggi University Hospital, Florence, Italy, 7 Pneumology and Intensive Care Unit, Careggi University Hospital, Florence, Italy, 8 Intensive Care Unit and Regional ECMO Referral Centre, Careggi University Hospital, Florence, Italy, 9 Infectious and Tropical Diseases Unit, Careggi University Hospital, Florence, Italy, $10 \mathrm{Imm}$ unology and Cell Therapy Unit, Careggi University Hospital, Florence, Italy, 11 Flow Cytometry Diagnostic Center and Immunoterapy (CDCl), Careggi University Hospital, Florence, Italy

๑ These authors contributed equally to this work.

* francesco.annunziato@unifi.it (FA); turano@ cerm.unifi.it (PT)

\section{Abstract}

The current pandemic emergence of novel coronavirus disease (COVID-19) poses a relevant threat to global health. SARS-CoV-2 infection is characterized by a wide range of clinical manifestations, ranging from absence of symptoms to severe forms that need intensive care treatment. Here, plasma-EDTA samples of 30 patients compared with age- and sexmatched controls were analyzed via untargeted nuclear magnetic resonance (NMR)-based metabolomics and lipidomics. With the same approach, the effect of tocilizumab administration was evaluated in a subset of patients. Despite the heterogeneity of the clinical symptoms, COVID-19 patients are characterized by common plasma metabolomic and lipidomic signatures ( $91.7 \%$ and $87.5 \%$ accuracy, respectively, when compared to controls). Tocilizumab treatment resulted in at least partial reversion of the metabolic alterations due to SARS-CoV-2 infection. In conclusion, NMR-based metabolomic and lipidomic profiling provides novel insights into the pathophysiological mechanism of human response to SARSCoV-2 infection and to monitor treatment outcomes.

\section{Author summary}

The current COVID-19 pandemic caused by severe acute respiratory syndrome coronavirus-2 (SARS-CoV-2) is markedly affecting the world population. Here we report about the small-molecule profile of patients hospitalized during the first wave of the COVID-19 pandemic in Florence (Italy). Using magnetic resonance spectroscopy, we showed that the 
"Excellence Departments 2018-2022 Project". The funders had no role in study design, data collection and analysis, decision to publish, or preparation of the manuscript.

Competing interests: The authors have declared that no competing interests exist. infection induces profound changes in the metabolome. The analysis of the specific metabolite changes and correlations with clinical data enabled the identification of potential biochemical determinants of the disease fingerprint. We also followed how metabolic alterations revert towards those of the control group upon treatment with tocilizumab, a recombinant humanized monoclonal antibody against the interleukin-6 receptor. These results open up possibilities for the monitoring of novel patients and their individual response to treatment.

\section{Introduction}

The World Health Organization announced coronavirus disease 2019 (COVID-19) outbreak a pandemic in March 2020 [1,2]. At the beginning of October 2020 over thirty-four millions of patients have been diagnosed by SARS-CoV-2 infection and about 1 million deaths are reported all over the world [3]. The SARS-CoV-2 infection is characterized by a wide range of clinical manifestations, ranging from absence of symptoms to severe forms that need intensive care treatment. About $20 \%$ of patients, particularly the older ones and those affected by chronic comorbidities such as hypertension, diabetes mellitus, renal and heart diseases, may develop interstitial pneumonia and respiratory distress requiring oxygen therapy or mechanical ventilation [4]. In addition to interstitial pneumonia and acute respiratory distress syndrome (ARDS), COVID-19 is associated with other life-threatening complications such as sepsis, thromboembolism and multi-organ failure [5]. Patients with the highest rate of morbidity and mortality following SARS-CoV-2 infection develop a hyperinflammatory syndrome due to the overproduction of early response proinflammatory cytokines (such as IL-1 $\beta$, IL-6, TNF $\alpha$, MCP-1)-the so called "cytokine storm"-leading to an increased vascular permeability, activation of coagulation pathways, dysregulation of T cells with associated lymphopenia, multiorgan injury and rapid clinical deterioration [6-9].

Metabolomics and lipidomics can contribute a system level picture, thus expanding the options that chemists can explore to help fight the pandemic [10]. The human metabolome is composed by an ensemble of several thousands of small molecules $(<1500-2000 \mathrm{Da})$ present on a very ample range of concentrations (from $<1 \mathrm{nM}$ to $>1 \mu \mathrm{M}$ ) and produced by the genome of the host organism and by the genomes of its microflora, as well as deriving from exogenous factors like medical treatments [11]. Blood plasma is a primary carrier of small molecules in the body, the relative concentrations of which reflect the physio-pathological state of the organism and thus possible tissue lesions and organ dysfunctions. The overall picture is complemented by alterations in the lipid components [12]. As a consequence, metabolomics and lipidomics of serum and plasma are increasingly used for successful patient stratification in various diseases [13-18]. Herein, a strong metabolomic and lipidomic signature of COVID19 is revealed via untargeted nuclear magnetic resonance (NMR) spectroscopy of plasmaEDTA $[19,20]$ from 30 patients compared with age- and sex-matched controls. Moreover, in a subset of patients, the metabolic effects due to tocilizumab administration were successfully investigated. This study had no sample-size calculation; the analysis included all patients who were admitted at the Infective and Tropical Diseases and at the Intensive Care Unit of the Careggi University Hospital, Florence (IT), in the period between March 10 and March 30 2020, before the rapid decline of hospitalizations for COVID-19.

\section{Results and discussion}

We analyzed via ${ }^{1} \mathrm{H}$ NMR spectroscopy the metabolomic and lipidomic profiles of plasmaEDTA samples obtained from 30 patients affected by COVID-19. SARS-CoV-2 infection was 
confirmed by positive RT-PCR on nasopharyngeal swab specimens. The plasma-EDTA samples available for the metabolomic analysis were collected between 2-23 days from clinical onset (mean 9 days). Samples from 30 non COVID-19 subjects, one-to-one matched for age and sex, were used as control group (CTR). Tocilizumab, a humanized anti-IL-6 receptor monoclonal antibody, was administered to 8 of the 30 COVID-19 patients enrolled and another plasma-EDTA sample for each patient was collected after 2-18 days of treatment (mean 5 days). Demographic and clinical characteristics of enrolled patients are reported in S1 Table. Our analyses considered 21 quantified metabolites and 114 lipoprotein-related parameters [21]. Lipoprotein quantification of plasma samples of two COVID-19 patients (COVID19-025 and COVID-19-027) was not possible for the presence of an interfering signal in the spectra, thus also their respective matched controls (CTR-4 and CTR-7) were removed from the lipoprotein analyses.

No outliers were identified using principal component analysis (PCA) on the entire population, both for metabolite and lipoprotein profiles (S1 Fig).

Plasma metabolite and lipoprotein profiles of COVID-19 patients and CTRs were compared using the Random Forest (RF) algorithm. The eight samples collected post-tocilizumab treatment are not included in these analyses. The RF model built on metabolite concentrations shows a significant differential clustering with $91.7 \%$ accuracy, $93.3 \%$ sensitivity, and $90.0 \%$ specificity (Fig 1A and $1 \mathrm{C}$ and S2A and S2 Table). In particular, a panel of 11 metabolites (Fig $1 \mathrm{D}$ and $\mathrm{S} 3 \mathrm{Table}$ ) displays significant alterations between COVID-19 patients and CTRs. One of them, giving rise to a detectable multiplet in the region between 7.21-7.30 ppm has not been assigned and is referred as "unknown". However, even if this signal is removed from the statistical model, the discrimination accuracy between COVID-19 patients and CTRs does not change significantly.

The RF model built on lipoprotein-related parameters shows a significant differential clustering with $87.5 \%$ accuracy, $85.7 \%$ sensitivity, and $89.3 \%$ specificity (Fig $1 \mathrm{~B}$ and $1 \mathrm{C}$ and S2B and S2 Table). Forty-eight features (Fig 1E and S4 Table) display significant alterations between COVID-19 patients and CTRs. These results demonstrate that COVID-19 patients are characterized by higher levels of VLDL particles, and lower levels of Apo A1, Apo A2, cholesterol and free-cholesterol HDL and LDL subfractions. In particular, the subfractions HDL3, HDL-4, LDL-4, LDL-5 of cholesterol are the most affected.

Correlations between clinical and metabolomic parameters were calculated and the results are reported in Fig 2.

Phenylalanine significantly correlates with C-reactive protein (CRP) and interleukin-6. Inflammation and immune activation impair the conversion of phenylalanine to tyrosine, as observed in patients suffering from sepsis, cancer, or HIV-1 infection [22-25]; accordingly, we found higher phenylalanine levels and a trend in lower tyrosine amounts in patients than in controls. Interestingly, a positive correlation between phenylalanine/tyrosine ratio and high CRP levels, has been already described by Murr and colleagues [26] in patients affected by coronary artery disease (CAD). These data are in accordance with ours, since SARS-CoV-2 infection is characterized not only by immune activation and systemic phlogosis, but also by microvascular endothelial damage and activation of coagulative cascade, as happens in CAD. Alveolar-arterial $\mathrm{O}_{2}$ gradient anticorrelates with citrate, accordingly the partial pressure of arterial oxygen and fraction of inspired oxygen $\left(\mathrm{PaO}_{2} / \mathrm{FiO}_{2}\right)$ ratio (known as Horowitz index) and the ratio between oxygen saturation and fraction of inspired oxygen $\left(\mathrm{SaO}_{2} / \mathrm{FiO}_{2}\right)$ positively correlates with citrate. This metabolite is known for its anti-oxidative, anti-coagulant and anti-inflammatory properties [27-29]. SARS-CoV-2 infection can cause lung damage, leading to ARDS, due not only to alveolar damage but also to diffuse microvascular endothelial damage and clot activation, mainly driven by pro-inflammatory cytokines, including IL-6 
A

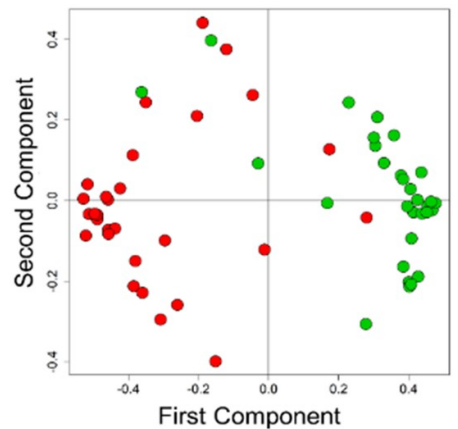

D

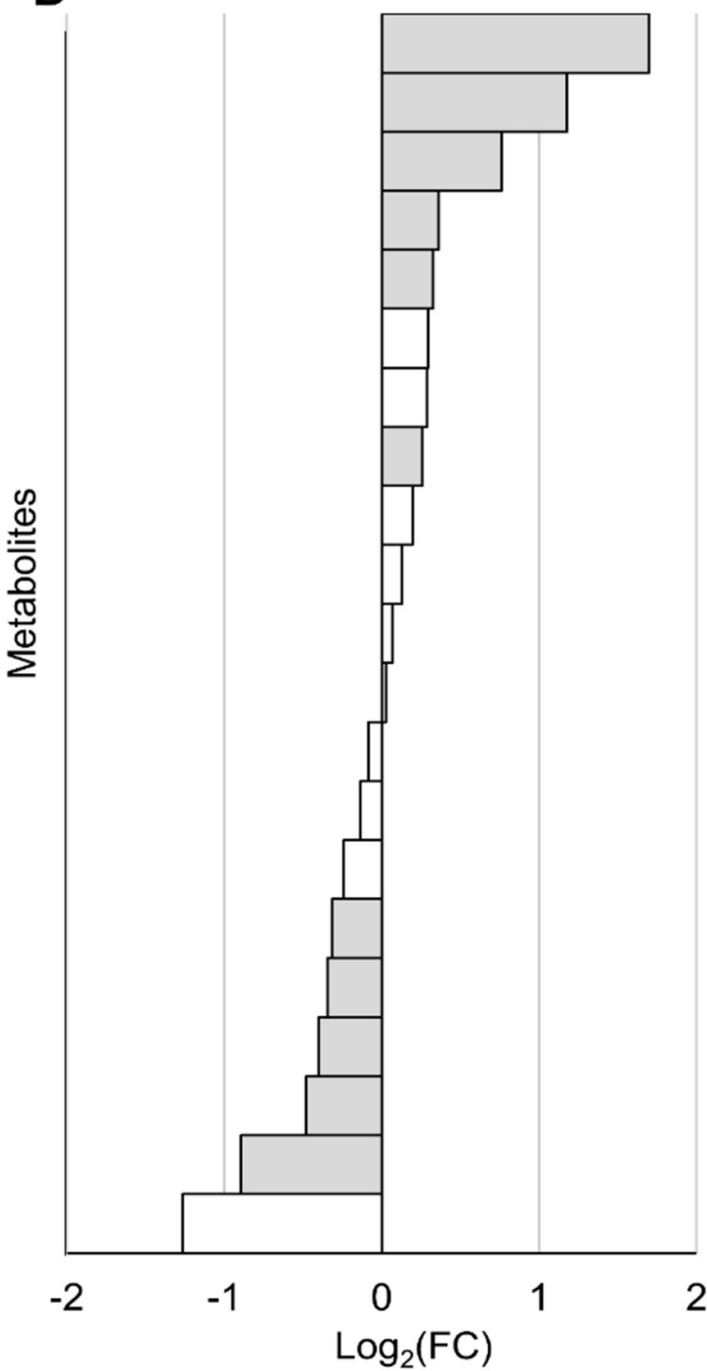

C

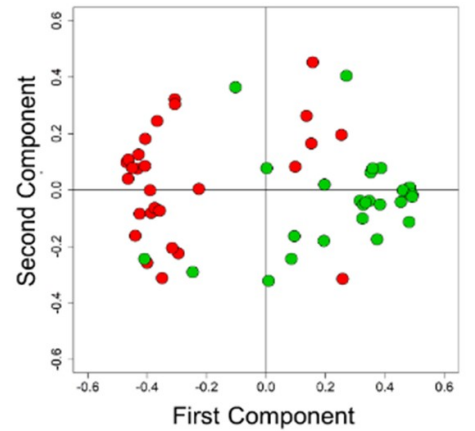

3-hydroxybutyrate

Mannose

Unknown

Pyruvate

Glycoproteins

Isoleucine

Leucine

Phenylalanine

Creatinine

Glucose

Valine

Lactate

Tyrosine

Formate

Acetate

Histidine

Glutamine

Glycine

Alanine

Citrate

Creatine

E

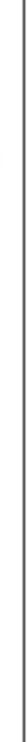

\begin{tabular}{rcc|cc}
\hline & \multicolumn{2}{c|}{ metabolites } & \multicolumn{2}{c}{ lipoproteins } \\
\hline & COVID-19 & CTR & COVID-19 & CTR \\
COVID-19 & 28 & 2 & 24 & 4 \\
CTR & 3 & 27 & 3 & 25 \\
\hline Predictive accuracy: & $91.7 \%$ & & \multicolumn{2}{|c|}{$87.5 \%$} \\
\hline
\end{tabular}
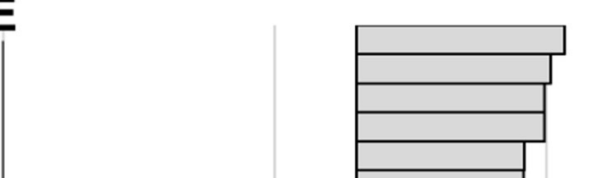

Tg-VLDL5

Tg-VLDL2

Tg-LDL1

Free Chol-VLDL2

Tg-VLDL4

Tg-LDL2

Tg-VLDL3

Tg-LDL

VLDL (Particle number)

Apo B VLDL

Tg-LDL4

Tg-VLDL

Tg-LDL3

Ph-VLDL4

$\mathrm{Tg}$

Apo A1/Apo B100

Tg-LDL6

Apo B LDL

LDL (Particle number)

Tg-HDL4

Apo A1 HDL3

Apo A1 HDL2

Chol-HDL3

$\mathrm{Ph}-\mathrm{HDL}$

Apo A2 HDL

Apo B LDL5

LDL5 (Particle number)

Chol-LDL1

Apo A2

$\mathrm{Ph}$-HDL3

Free Chol-LDL5

Chol

Free Chol-LDL

Apo A1

Ph-LDL

Apo A1-HDL

Chol-HDL

Ph-LDL5

Apo A1-HDL4

Apo A2-HDL4

Ph-HDL4

Chol-LDL

Chol-HDL4

Free Chol-HDL4

Free Chol-HDL3

Chol-LDL5

Ph-LDL4

Chol-LDL4

Fig 1. Metabolomic/lipidomic alterations in COVID-19 patients. (A-B) Proximity plots of the RF model discriminating COVID-19 patients (red dots), and CTR subjects (green dots) using A) the 21 quantified metabolites and B) the lipoprotein-related parameters. (C) Confusion matrices with predictive accuracy values of each model. (D) Values of $\log _{2}$ Fold Change (FC) of quantified metabolites. Grey bars represent p-values $<0.05$ after FDR correction. (E) Values of Log 2 (FC) of lipoproteinrelated parameters significantly different (p-value $<0.05$ after FDR correction) between COVID-19 patients and controls. Metabolites/lipoproteins with $\log _{2}(\mathrm{FC})$ positive/negative values have higher/lower concentration in plasma samples from COVID-19 patients with respect to controls.

https://doi.org/10.1371/journal.ppat.1009243.g001 


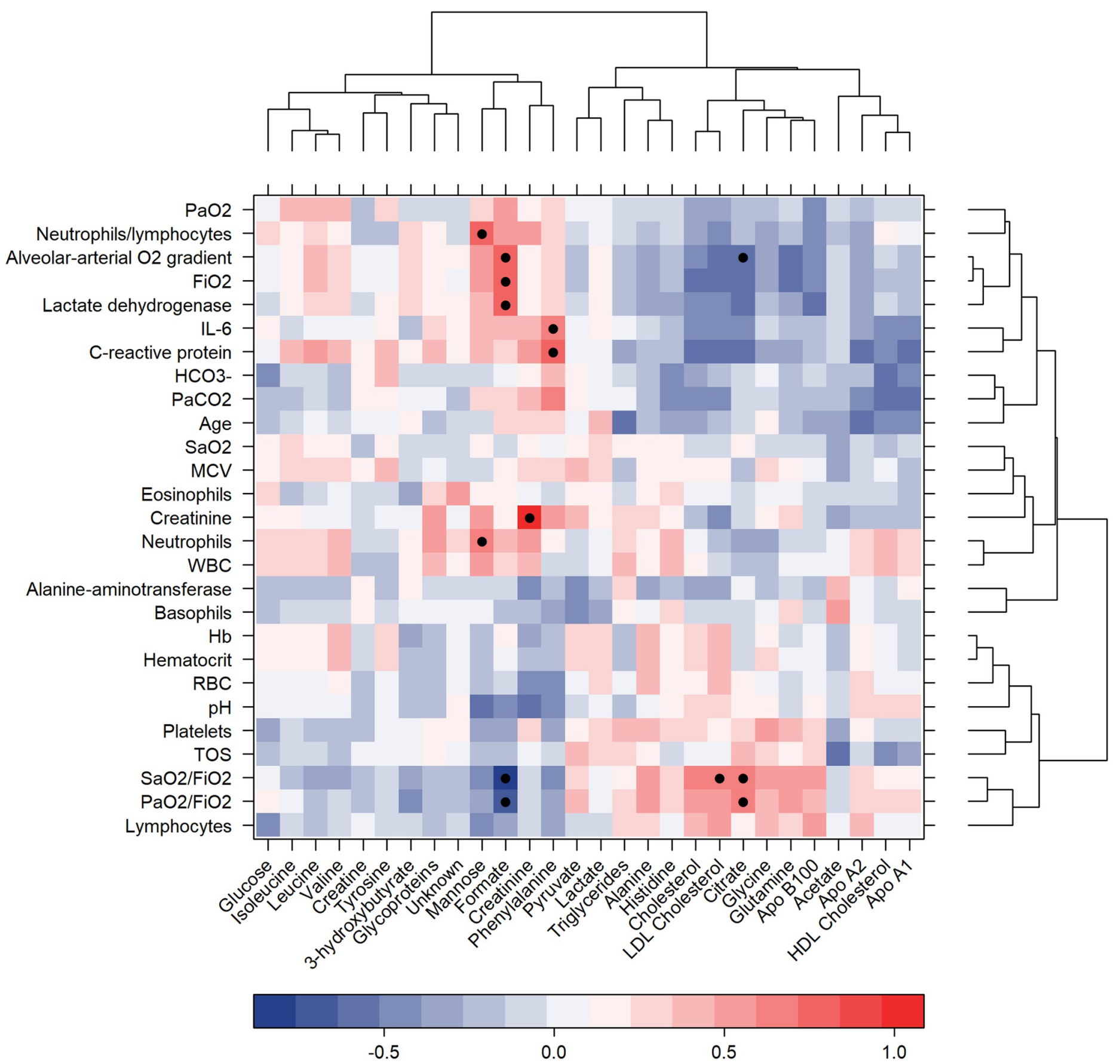

Fig 2. Heatmap correlations between clinical and metabolomic parameters. R values are shown as different degree of color intensity (red, positive correlations; blue, negative correlation). $\mathrm{R}$ values are reported in the plot only for statistically significant correlations ( $\mathrm{p}$-value $<0.05$ after FDR correction).

$[30,31]$. The protective role of citrate on endothelial integrity was recently reported by Dellepiane and colleagues [32]. Moreover, the consumption of citrate and other carboxylates is promoted by hypoxic conditions in red blood cells [33].

Formate shows the inverse pattern of correlation with respect to citrate, and it significantly correlates also with lactate dehydrogenase and $\mathrm{FiO}_{2}$. Regarding lipoprotein-related parameters only LDL cholesterol significantly correlates with the $\mathrm{SaO}_{2} / \mathrm{FiO}_{2}$ ratio. 
In line with these observations, metabolites provide an optimal discrimination (accuracy 90.0\%, 100.0\% sensitivity, 83.3\% specificity) between COVID-19 patients treated and nontreated with invasive ventilation (S3A and S3B Fig), with formate and citrate as the most important features of the model. Instead, no significant clustering is present in the model calculated with lipoprotein-related parameters (S3C and S3D Fig).

Despite plasma samples of COVID-19 patients are characterized by higher levels of VLDL and associated triglycerides, we observed a general reduction of HDL and LDL cholesterolrelated parameters. Downregulation of lipids in COVID-19 blood sera has already been observed [34-37] and it has been hypothesized that lipids (in particular cholesterol and fatty acids) could play a pivotal role in virus replication and assembly $[38,39]$. Our data suggest that only LDL and HDL could be implied in this mechanism.

Accordingly, in a recent study, LDL and HDL levels were inversely correlated to disease severity and poor prognosis [40]. Furthermore, overproduction of VLDL has been linked with the processes inducing insulin resistance in COVID-19 patients [35].

We also detected an accumulation of mannose in the plasma of COVID-19 patients and a significant positive correlation between plasma mannose levels and neutrophils and between mannose and the neutrophils to lymphocytes ratio. An increment of mannose could be related to different reasons: it could be associated to its binding to lectin in order to promote complement activation [34], or it could be linked to insulin resistance. Indeed, plasma mannose levels are elevated in subjects with insulin resistance independently of obesity [41] and there are increasing evidences that a bidirectional relationship between COVID-19 and diabetes exists [42].

The increment of pyruvate and 3-hydroxybutyrate, along with the strong decrement of citrate and free amino acids (alanine, glycine, glutamine, histidine) in plasma of COVID-19 patients can be ascribed to an impairment of the energetic metabolism. Indeed, during inflammatory states amino acids can be used to provide energy and materials for the proliferation and phagocytosis of immune cells. It is important to underline that pyruvate is a metabolite particularly sensitive to pre-analytical procedures, thus further investigations to confirm its alteration are needed $[43,44]$.

Among the 30 COVID-19 patients enrolled, 18 patients presented ARDS. Thus, the possibility that ARDS could significantly alter the profile of COVID-19 was examined. The RF models calculated both on metabolites and lipoprotein-related parameters can only slightly cluster ARDS patients with respect to the other COVID-19 patients (metabolite model: accuracy $76.7 \%, 88.9 \%$ sensitivity, $58.3 \%$ specificity; lipoprotein model: accuracy $75.0 \%, 81.2 \%$ sensitivity, $66.7 \%$ specificity). These results demonstrate that the metabolomic profile of COVID19 patients is mainly dictated by the pathology or by the host response to the virus infection, rather than by the concomitant presence of ARDS.

Multilevel partial least square discriminant analysis (mPLS-DA) was used to analyze NMR data of pre- and post-tocilizumab samples in a pairwise multivariate fashion. The mPLS-DA model built on metabolites shows significant differential clustering, yielding a discrimination accuracy of $80.3 \%$ (Fig 3A). The two pairs of samples collected from patients who died (COVID19-020 and COVID-19-021) present the smallest shift within the metabolomic subspace. The same trend is observed for COVID-19-018 patient who, unfortunately, was transferred to another hospital and no follow-up and outcome information was available. Univariate analysis enables the identification of a panel of eight metabolites (Fig 3B-3I and S5 Table) significantly different (before FDR correction) between pre- and post-tocilizumab patients. The post-treatment levels of these metabolites partially or completely revert towards the levels of CTR subjects.

The mPLS-DA model built using lipoprotein-related parameters shows a significant discrimination between samples collected pre- and post-tocilizumab treatment (accuracy of 

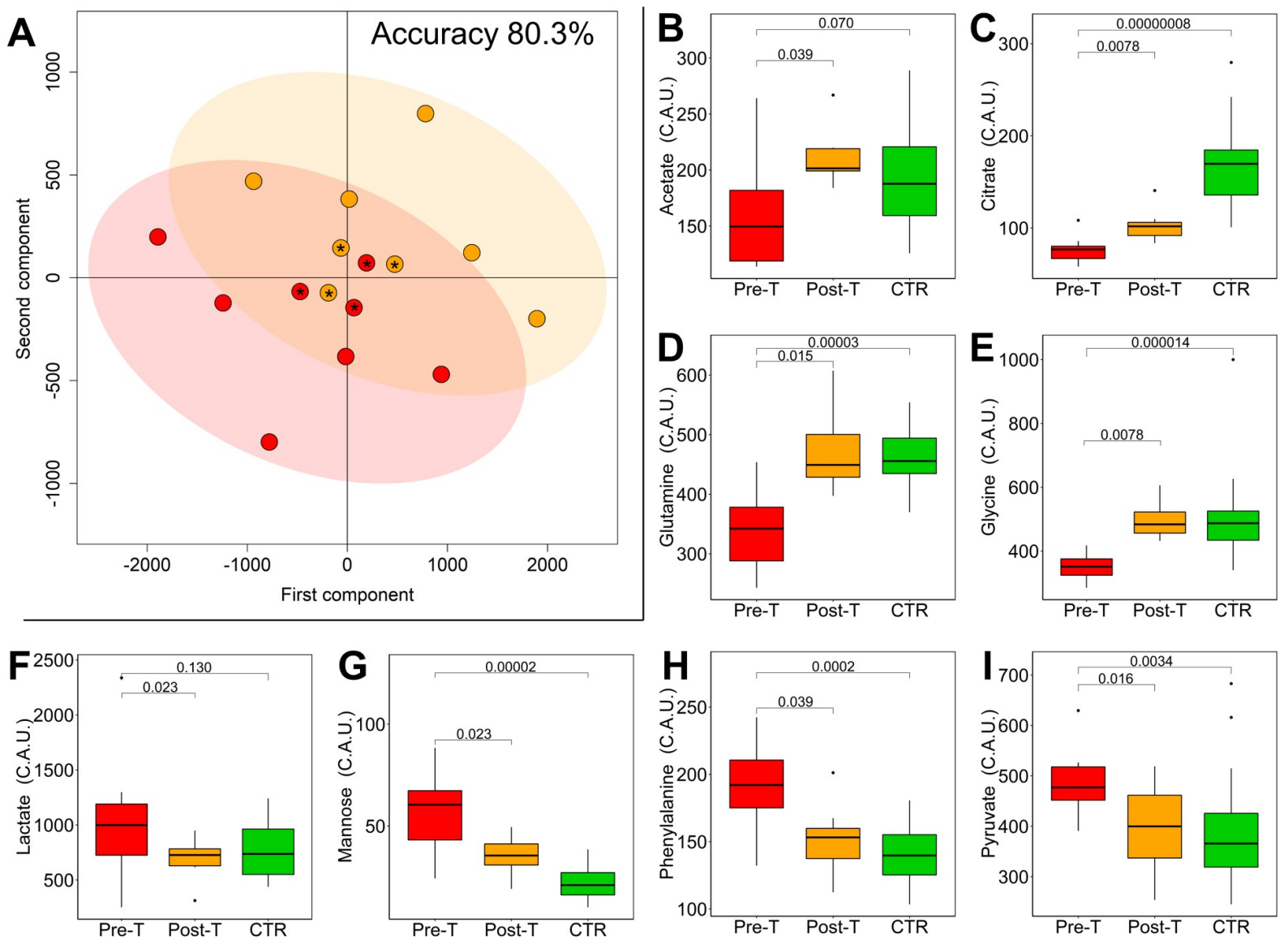

Fig 3. Tocilizumab treatment reverts metabolomic/lipidomic alterations in COVID-19 patients. (A) Score plot (of the first two principal components) and accuracy of the mPLS-DA model discriminating COVID-19 patients at pre- (red dots) and post- (orange dots) tocilizumab treatment using the 21 quantified metabolites. Patients 18, 20 and 21 are marked with *. (B-I) Boxplots of the statistically significant metabolites discriminating of pre- (red) and post- (orange) tocilizumab samples, p-values obtained using Wilcoxon signed-rank test are also reported. Boxplots of controls (green) and the p-values (Wilcoxon-Mann-Whitney test) for the comparison between pre-treatment and CTR are reported. P-values adjusted for FDR are reported in S5 Table.

https://doi.org/10.1371/journal.ppat.1009243.g003

82.8\%) (S4 Fig). Univariate analysis identifies 19 lipoprotein parameters (S6 Table) significantly different between pre- and post-tocilizumab treatment. In particular, HDL-1 subfractions of cholesterol, phospholipids, and Apo A2 showed lower levels at post-treatment, whereas LDL-5, HDL-4, IDL, VLDL-1, and VLDL-2 of many subfractions are higher at posttreatment. The general increment of lipoprotein subfractions after treatment confirms the metabolic reversion and supports the key role of lipids in the metabolism of COVID-19 patients.

In summary, in this study a strong plasma metabolomic and lipidomic signature of SarsCoV-2 infection is identified, in agreement with other studies where NMR or mass spectrometry have been used to study cohorts of COVID-19 patients from different countries and characterized by different degrees of severity/clinical manifestations [34-37]. Although the two analytical platforms do not address the same sets of molecules, common metabolic dysfunctions emerge from the comparison of all these studies, which include lipid metabolism, protein glycosylation and amino acid metabolism. Correlations between clinical parameters and some metabolites are shown, which include mannose and phenylalanine levels. In addition, here we found that some molecules, whose levels correlate with alveolar-capillary membrane injury are 
affected by mechanical ventilation. Of note, in the case of patients who underwent tocilizumab treatment, metabolic alterations revert towards those of the control group.

\section{Materials and methods}

\section{Ethics statement}

The study was approved by the Comitato Etico Regionale- sezione area vasta centro (protocol 16859) and it complies with the 1964 Declaration of Helsinki and its later amendments. Written informed consent was obtained from recruited patients.

\section{Patients characteristics}

In the period between March 10 and March 30, 2020 we enrolled 30 COVID-19 patients that were admitted at the Infective and Tropical Diseases Unit and at the Intensive Care Unit of the Careggi University Hospital, Florence, Italy. All patients were of Caucasian ethnicity. Demographic and clinical features of the enrolled patients are summarized in S1 Table.

\section{Plasma sample preparation}

Plasma samples were collected from all the subjects enrolled in the study, according to standard procedures $[43,44]$. Blood samples were collected in ethylene diamine tetra-acetate (EDTA)-coated blood collection tubes and stored at room temperature. Ficoll, a neutral highly branched polymer formed by the co-polymerization of sucrose and epichlorohydrin, was used for blood separation. $14 \mathrm{~mL}$ of blood were gently placed in $25 \mathrm{~mL}$ tubes containing $9 \mathrm{~mL}$ of Ficoll. Tubes were centrifugated $1500 \mathrm{~g}$ for 20 minutes. Plasma was collected and rapidly stored in a $-20^{\circ} \mathrm{C}$ freezer pending NMR analysis.

\section{NMR sample preparation, spectra processing and spectral analysis}

NMR samples were prepared according to standard procedures [19,20,45]. NMR spectra for all samples were acquired using a Bruker $600 \mathrm{MHz}$ spectrometer (Bruker BioSpin) operating at $600.13 \mathrm{MHz}$ proton Larmor frequency and equipped with a $5 \mathrm{~mm}$ PATXI ${ }^{1} \mathrm{H}_{-}{ }^{13} \mathrm{C}_{-}^{-15} \mathrm{~N}$ and ${ }^{2} \mathrm{H}$-decoupling probe including a $\mathrm{z}$-axis gradient coil, an automatic tuning-matching (ATM) and an automatic refrigerated sample changer (SampleJet, Bruker BioSpin). A BTO 2000 thermocouple served for temperature stabilization at the level of approximately $0.1 \mathrm{~K}$ of the sample. Before measurement, to equilibrate temperature at $310 \mathrm{~K}$, samples were kept for at least 5 minutes inside the NMR probe head.

For each plasma sample, two one-dimensional ${ }^{1} \mathrm{H}$ NMR spectra were acquired with water peak suppression and different pulse sequences that allowed the selective detection of different molecular components. The spectra were: 1) a standard NOESY using 32 scans, 98,304 data points, a spectral width of $18,028 \mathrm{~Hz}$, an acquisition time of $2.7 \mathrm{~s}$, a relaxation delay of $4 \mathrm{~s}$ and a mixing time of $0.01 \mathrm{~s}$. This kind of spectrum is made up of signals arising from low molecular weight molecules (metabolites) and signals arising from macromolecules such as lipoproteins and lipids; 2) a standard spin echo Carr-Purcell-Meiboom-Gill (CPMG) using 32 scans, 73,728 data points, a spectral width of $12,019 \mathrm{~Hz}$ and a relaxation delay of $4 \mathrm{~s}$. This NMR sequence allows the selective detection of signals arising only from low molecular weight metabolites.

Before applying Fourier transform, free induction decays were multiplied by an exponential function equivalent to a $0.3 \mathrm{~Hz}$ line-broadening factor. Transformed spectra were automatically corrected for phase and baseline distortions and calibrated to the anomeric glucose doubled at $\delta 5.24$ ppm, using TopSpin 3.6.2 (Bruker BioSpin) [19,20]. 


\section{Statistical analysis}

All data analyses were performed using the " $R$ " statistical environment. Metabolites, whose peaks in the CPMG spectra were well defined and resolved, were assigned and their concentrations analyzed. The assignment procedure was performed using an ${ }^{1} \mathrm{H}$ NMR spectra library of pure organic compounds (BBIOREFCODE, Bruker BioSpin), public databases, e.g. Human Metabolome Database [11], storing reference ${ }^{1} \mathrm{H}$ NMR spectra of metabolites, and using literature data when available. The spectral regions associated to the 21 assigned metabolites (S7 Table) were integrated using a R script in-house developed. Quantification of 114 lipid main fractions and subfractions was performed using the Bruker IVDr platform [21].

Both metabolites and lipoprotein-related parameters were analyzed via multivariate analysis. Unsupervised Principal Component Analysis (PCA) was used as first exploratory analysis to obtain a preliminary data outlook (i.e. cluster detection and screening for outliers). The Random Forest (RF) algorithm [46] was used for classification in the comparison between COVID-19 patients and CTR. RF is a classification algorithm that uses an ensemble of unpruned decision trees (forest), each of which is built on a bootstrap sample of the training data using a randomly selected subset of variables (metabolites or lipoprotein-related parameters) $[47,48]$. The percentage of trees in the forest that assign one sample to a specific class can be inferred as a probability of belonging to a given class [13]. In our case, each tree was used to predict whether a sample represents a COVID-19 patient or a CTR subject. Because the outof-bag (OOB) observations were not used in the fitting of the trees, the OOB estimates are cross-validated, accuracy estimates, and represent an unbiased estimation of the generalization error [46]. Accuracy, sensitivity, and specificity of all calculated models were assessed according to the standard definitions. For all calculations, the R package 'Random Forest' [46] was used to grow a forest of 1000 trees, using the default settings.

Pairwise comparisons between pre- and post-treatment samples were performed using multilevel Partial Least Square Discriminant Analysis (mPLS-DA) and results validated using a Monte Carlo Cross-Validation scheme (MCCV, script in house developed): each dataset was randomly divided by 1000 times into a training set ( $80 \%$ of the data) which was used to build the model and a test set ( $20 \%$ of the data) which was used to test the integrity of the model. Accuracy, sensitivity, and specificity of all calculated models were assessed according to the standard definitions.

On the biological assumption that metabolite and lipoprotein concentrations are not normally distributed, non-parametric tests were used for the univariate analysis. WilcoxonMann-Whitney test was used to infer differences between the metabolite/lipid levels in the comparison between COVID-19 patients and CTR. Instead for pairwise comparison between pre- and post-treatment samples the Wilcoxon signed-rank test was utilized [49]. P-values were adjusted for multiple testing using the false discovery rate (FDR) procedure with Benjamini-Hochberg [50] correction at $\alpha=0.05$. The effect size (Ef) was also calculated [51] to aid in the identification of the meaningful signals giving an estimation of the magnitude of the separation between the different groups. The magnitude is assessed using the thresholds provided in Romano et al. [52], i.e. $|\mathrm{Ef}|<0.147$ "negligible", $|\mathrm{Ef}|<0.33$ "small", $|\mathrm{Ef}|<0.474$ "medium", otherwise "large". Pearson correlations, adjusted for FDR using BH methods, were also calculated.

\section{Supporting information}

S1 Fig. PCA analysis. Score plots (PC1 vs. PC2) of the unsupervised PCA model of A) 21 quantified metabolites, B) lipoprotein-related parameters; COVID-19 patients (red dots); CTR 
subjects (green dots).

(TIF)

S2 Fig. Metabolomic/lipidomic alterations in COVID-19 patients. Variable importance plots of the Random Forest models discriminating COVID-19 patients and control subjects. A) 21 quantified metabolites, B) lipoprotein-related parameters.

S3 Fig. Alterations induced by invasive ventilation in COVID-19 patients. Proximity plot (of the first two principal components) and accuracy of the Random Forest model discriminating COVID-19 patients treated (blue dots) and non-treated (sea green dots) with invasive ventilation using metabolites (A) and lipoprotein-related parameters (C). Variable importance plots of the two Random Forest models: B) 21 quantified metabolites, D) lipoprotein-related parameters.

S4 Fig. Alterations in lipoprotein profile induced by Tocilizumab treatment. A) Score plot (of the first two principal components) and accuracy of the mPLS-DA model discriminating COVID-19 patients pre- (red dots) and post- (orange dots) tocilizumab treatment using the lipoprotein-related parameters.

S1 Table. Demographic and clinical characteristics of COVID-19 patients. ACF denotes acute cardiac failure, AI autoimmune disease, AKI acute kidney injury, ARDS acute respiratory distress syndrome, CKD chronic kidney disease, CVD cardiovascular disease, D deceased, DBT type 2 diabetes, DH discharged home, DYS dyslipidemia, $\mathrm{H}$ hypertension, $\mathrm{K}$ cancer, LTFU lost to follow-up. (XLSX)

S2 Table. Metabolomic/lipidomic alterations in COVID-19 patients: multivariate analysis. Random Forest scores of the model discriminating COVID-19 patients and controls using the 21 quantified metabolites and lipoprotein-related parameters. P: predicted class; S. numeric score (controls: $0<\mathrm{S}<0.5$; COVID-19 patients: $0.5<\mathrm{S}<1$ ). (XLSX)

S3 Table. Metabolomic alterations in COVID-19 patients: univariate analysis. Univariate analysis of the 21 quantified metabolites for the comparison between COVID-19 patients and control subjects. The median and MAD of each metabolite in the two groups are reported. The p-value of the univariate Wilcoxon-Mann-Whitney test together with the p-value calculated after false discovery rate correction and the effect size, using the Cliffs delta formulation, were also reported for each metabolite.

(XLSX)

S4 Table. Lipidomic alterations in COVID-19 patients: univariate analysis. Univariate analysis of the lipoprotein-related parameters for the comparison between COVID-19 patients and control subjects. The median and MAD of each parameter in the two groups are reported. The $\mathrm{p}$-value of the univariate Wilcoxon-Mann-Whitney test together with the p-value calculated after false discovery rate correction and the effect size, using the Cliffs delta formulation, were also reported for each parameter.

(XLSX)

S5 Table. Metabolomic alterations induced by Tocilizumab treatment: univariate analysis. Univariate analysis of the 21 quantified metabolites for the comparison between COVID-19 
patients before and after tocilizumab treatment. The p-value of the univariate WilcoxonSigned-Rack test together with the p-value calculated after false discovery rate correction and the effect size were reported for each metabolite.

(XLSX)

S6 Table. Lipidomic alterations induced by Tocilizumab treatment: univariate analysis. Univariate analysis of the lipoprotein-related parameters for the comparison between COVID-19 patients before and after tocilizumab treatment. The p-value of the univariate Wilcoxon-Signed-Rack test together with the $\mathrm{p}$-value calculated after false discovery rate correction and the effect size were reported for each parameter. (XLSX)

S7 Table. List of the metabolites assigned and analyzed in plasma samples. For each metabolite, the chemical shift of the signal used for the analysis and the corresponding multiplicity are provided; $\mathrm{d}$. $\mathrm{s}=$ singlet; $\mathrm{d}=$ doublet; $\mathrm{t}=$ triplet; $\mathrm{dd}=$ doublet of doublets; $\mathrm{m}=$ multiplet. The Human Metabolome Database (HMDB) compound ID of each metabolite is reported. (XLSX)

\section{Acknowledgments}

P.T., C.L., L.T., V.G., A.V. and G.M acknowledge the helpful discussions with our partners of the Metabolomics based COVID-19 Research Network (CV19 Research Network) and the support and the use of resources of Instruct-ERIC, a Landmark ESFRI project, and specifically the CERM/CIRMMP Italy Centre.

\section{Author Contributions}

Conceptualization: Francesco Annunziato, Paola Turano.

Data curation: Alessio Mazzoni, Lorenzo Salvati.

Formal analysis: Gaia Meoni, Veronica Ghini, Laura Maggi, Alessia Vignoli, Francesco Liotta, Lorenzo Cosmi.

Investigation: Gaia Meoni, Veronica Ghini, Alessia Vignoli.

Methodology: Gaia Meoni, Veronica Ghini, Alessia Vignoli.

Resources: Laura Maggi, Alessio Mazzoni, Lorenzo Salvati, Manuela Capone, Anna Vanni, Paolo Fontanari, Federico Lavorini, Adriano Peris, Alessandro Bartoloni, Claudio Luchinat.

Supervision: Claudio Luchinat, Francesco Annunziato, Paola Turano.

Writing - original draft: Gaia Meoni, Veronica Ghini, Laura Maggi, Alessia Vignoli, Francesco Liotta, Lorenzo Cosmi, Francesco Annunziato, Paola Turano.

Writing - review \& editing: Lorenzo Salvati, Leonardo Tenori, Claudio Luchinat.

\section{References}

1. WHO Director-General's opening remarks at the media briefing on COVID-19-2 October 2020. [cited 9 Oct 2020]. Available: https://www.who.int/dg/speeches/detail/who-director-general-s-opening-remarksat-the-media-briefing-on-covid-19-2-october-2020

2. Zhou P, Yang X-L, Wang X-G, Hu B, Zhang L, Zhang W, et al. A pneumonia outbreak associated with a new coronavirus of probable bat origin. Nature. 2020; 579: 270-273. https://doi.org/10.1038/s41586020-2012-7 PMID: 32015507 
3. Coronavirus Disease (COVID-19) Situation Reports. [cited 9 Oct 2020]. Available: https://www.who.int/ emergencies/diseases/novel-coronavirus-2019/situation-reports

4. Wu Z, McGoogan JM. Characteristics of and Important Lessons From the Coronavirus Disease 2019 (COVID-19) Outbreak in China: Summary of a Report of 72314 Cases From the Chinese Center for Disease Control and Prevention. JAMA. 2020; 323: 1239-1242. https://doi.org/10.1001/jama.2020. 2648 PMID: 32091533

5. Zhou F, Yu T, Du R, Fan G, Liu Y, Liu Z, et al. Clinical course and risk factors for mortality of adult inpatients with COVID-19 in Wuhan, China: a retrospective cohort study. Lancet (London, England). 2020; 395: 1054-1062. https://doi.org/10.1016/S0140-6736(20)30566-3 PMID: 32171076

6. Costela-Ruiz VJ, Illescas-Montes R, Puerta-Puerta JM, Ruiz C, Melguizo-Rodríguez L. SARS-CoV-2 infection: The role of cytokines in COVID-19 disease. Cytokine \& Growth Factor Reviews. 2020; 54: 62-75. https://doi.org/10.1016/j.cytogfr.2020.06.001 PMID: 32513566

7. Mazzoni A, Salvati L, Maggi L, Capone M, Vanni A, Spinicci M, et al. Impaired immune cell cytotoxicity in severe COVID-19 is IL-6 dependent. The Journal of Clinical Investigation. 2020; 130: 4694-4703. https://doi.org/10.1172/JCl138554 PMID: 32463803

8. Vultaggio A, Vivarelli E, Virgili G, Lucenteforte E, Bartoloni A, Nozzoli C, et al. Prompt Predicting of Early Clinical Deterioration of Moderate-to-Severe COVID-19 Patients: Usefulness of a Combined Score Using IL-6 in a Preliminary Study. J Allergy Clin Immunol Pract. 2020; 8: 2575-2581.e2. https:// doi.org/10.1016/j.jaip.2020.06.013 PMID: 32565226

9. Huang C, Wang Y, Li X, Ren L, Zhao J, Hu Y, et al. Clinical features of patients infected with 2019 novel coronavirus in Wuhan, China. The Lancet. 2020; 395: 497-506. https://doi.org/10.1016/S0140-6736 (20)30183-5 PMID: 31986264

10. Opatz T, Senn-Bilfinger J, Richert C. Thoughts on What Chemists Can Contribute to Fighting SARSCoV-2 -A Short Note on Hand Sanitizers, Drug Candidates and Outreach. Angew Chem Int Ed Engl. 2020; 59: 9236-9240. https://doi.org/10.1002/anie.202004721 PMID: 32329159

11. Wishart DS, Feunang YD, Marcu A, Guo AC, Liang K, Vázquez-Fresno R, et al. HMDB 4.0: the human metabolome database for 2018. Nucleic Acids Research. 2018; 46: D608-D617. https://doi.org/10. 1093/nar/gkx1089 PMID: 29140435

12. Yang K, Han X. Lipidomics: Techniques, Applications, and Outcomes Related to Biomedical Sciences. Trends in Biochemical Sciences. 2016; 41: 954-969. https://doi.org/10.1016/j.tibs.2016.08.010 PMID: 27663237

13. Vignoli A, Tenori L, Giusti B, Takis PG, Valente S, Carrabba N, et al. NMR-based metabolomics identifies patients at high risk of death within two years after acute myocardial infarction in the AMI-Florence II cohort. BMC medicine. 2019; 17: 3. https://doi.org/10.1186/s12916-018-1240-2 PMID: 30616610

14. Meoni G, Lorini S, Monti M, Madia F, Corti G, Luchinat C, et al. The metabolic fingerprints of HCV and HBV infections studied by Nuclear Magnetic Resonance Spectroscopy. Scientific Reports. 2019; 9: 4128. https://doi.org/10.1038/s41598-019-40028-4 PMID: 30858406

15. McCartney A, Vignoli A, Biganzoli L, Love R, Tenori L, Luchinat C, et al. Metabolomics in breast cancer: A decade in review. Cancer Treatment Reviews. 2018; 67: 88-96. https://doi.org/10.1016/j.ctrv.2018. 04.012 PMID: 29775779

16. Monnerie S, Comte B, Ziegler D, Morais JA, Pujos-Guillot E, Gaudreau P. Metabolomic and Lipidomic Signatures of Metabolic Syndrome and its Physiological Components in Adults: A Systematic Review. Scientific Reports. 2020; 10: 669. https://doi.org/10.1038/s41598-019-56909-7 PMID: 31959772

17. Bertini I, Cacciatore S, Jensen BV, Schou JV, Johansen JS, Kruhøffer M, et al. Metabolomic NMR Fingerprinting to Identify and Predict Survival of Patients with Metastatic Colorectal Cancer. Cancer Res. 2012; 72: 356-364. https://doi.org/10.1158/0008-5472.CAN-11-1543 PMID: 22080567

18. Zhang $L$, Zhu B, Zeng $Y$, Shen $H$, Zhang J, Wang X. Clinical lipidomics in understanding of lung cancer: Opportunity and challenge. Cancer Letters. 2020; 470: 75-83. https://doi.org/10.1016/j.canlet.2019.08. 014 PMID: 31655086

19. Vignoli A, Ghini V, Meoni G, Licari C, Takis PG, Tenori L, et al. High-Throughput Metabolomics by 1D NMR. Angew Chem Int Ed Engl. 2019; 58: 968-994. https://doi.org/10.1002/anie.201804736 PMID: 29999221

20. Takis PG, Ghini V, Tenori L, Turano P, Luchinat $\mathrm{C}$. Uniqueness of the NMR approach to metabolomics. TrAC Trends in Analytical Chemistry. 2019; 120: 115300. https://doi.org/10.1016/j.trac.2018.10.036

21. Jiménez B, Holmes E, Heude C, Tolson RF, Harvey N, Lodge SL, et al. Quantitative Lipoprotein Subclass and Low Molecular Weight Metabolite Analysis in Human Serum and Plasma by 1H NMR Spectroscopy in a Multilaboratory Trial. Anal Chem. 2018; 90: 11962-11971. https://doi.org/10.1021/acs. analchem.8b02412 PMID: 30211542 
22. Neurauter G, Schröcksnadel K, Scholl-Bürgi S, Sperner-Unterweger B, Schubert C, Ledochowski M, et al. Chronic immune stimulation correlates with reduced phenylalanine turnover. Curr Drug Metab. 2008; 9: 622-627. https://doi.org/10.2174/138920008785821738 PMID: 18781914

23. Ploder M, Neurauter G, Spittler A, Schroecksnadel K, Roth E, Fuchs D. Serum phenylalanine in patients post trauma and with sepsis correlate to neopterin concentrations. Amino Acids. 2008; 35: 303-307. https://doi.org/10.1007/s00726-007-0625-x PMID: 18163176

24. Zangerle R, Kurz K, Neurauter G, Kitchen M, Sarcletti M, Fuchs D. Increased blood phenylalanine to tyrosine ratio in HIV-1 infection and correction following effective antiretroviral therapy. Brain Behav Immun. 2010; 24: 403-408. https://doi.org/10.1016/j.bbi.2009.11.004 PMID: 19925861

25. Capuron L, Schroecksnadel S, Féart C, Aubert A, Higueret D, Barberger-Gateau P, et al. Chronic lowgrade inflammation in elderly persons is associated with altered tryptophan and tyrosine metabolism: role in neuropsychiatric symptoms. Biol Psychiatry. 2011; 70: 175-182. https://doi.org/10.1016/j. biopsych.2010.12.006 PMID: 21277567

26. Murr C, Grammer TB, Meinitzer A, Kleber ME, März W, Fuchs D. Immune activation and inflammation in patients with cardiovascular disease are associated with higher phenylalanine to tyrosine ratios: the ludwigshafen risk and cardiovascular health study. J Amino Acids. 2014; 2014: 783730. https://doi.org/ 10.1155/2014/783730 PMID: 24660059

27. Grundström G, Christensson A, Alquist M, Nilsson L-G, Segelmark M. Replacement of acetate with citrate in dialysis fluid: a randomized clinical trial of short term safety and fluid biocompatibility. BMC Nephrol. 2013; 14: 216. https://doi.org/10.1186/1471-2369-14-216 PMID: 24103587

28. Gabutti L, Lucchini B, Marone C, Alberio L, Burnier M. Citrate- vs. acetate-based dialysate in bicarbonate haemodialysis: consequences on haemodynamics, coagulation, acid-base status, and electrolytes. BMC Nephrol. 2009; 10: 7. https://doi.org/10.1186/1471-2369-10-7 PMID: 19265544

29. Marengo M, Dellepiane S, Cantaluppi V. Extracorporeal Treatments in Patients with Acute Kidney Injury and Sepsis. Contrib Nephrol. 2017; 190: 1-18. https://doi.org/10.1159/000468912 PMID: 28535515

30. Cipolloni L, Sessa F, Bertozzi G, Baldari B, Cantatore S, Testi R, et al. Preliminary Post-Mortem COVID-19 Evidence of Endothelial Injury and Factor VIII Hyperexpression. Diagnostics (Basel). 2020; 10. https://doi.org/10.3390/diagnostics10080575 PMID: 32784826

31. Lai C-C, Shih T-P, Ko W-C, Tang H-J, Hsueh P-R. Severe acute respiratory syndrome coronavirus 2 (SARS-CoV-2) and coronavirus disease-2019 (COVID-19): The epidemic and the challenges. Int J Antimicrob Agents. 2020; 55: 105924. https://doi.org/10.1016/j.ijantimicag.2020.105924 PMID: 32081636

32. Dellepiane S, Medica D, Guarena C, Musso T, Quercia AD, Leonardi G, et al. Citrate anion improves chronic dialysis efficacy, reduces systemic inflammation and prevents Chemerin-mediated microvascular injury. Scientific Reports. 2019; 9: 10622. https://doi.org/10.1038/s41598-019-47040-8 PMID: 31337804

33. Nemkov T, Sun K, Reisz JA, Yoshida T, Dunham A, Wen EY, et al. Metabolism of Citrate and Other Carboxylic Acids in Erythrocytes As a Function of Oxygen Saturation and Refrigerated Storage. Front Med. 2017; 4. https://doi.org/10.3389/fmed.2017.00175 PMID: 29090212

34. Shen B, Yi X, Sun Y, Bi X, Du J, Zhang C, et al. Proteomic and Metabolomic Characterization of COVID-19 Patient Sera. Cell. 2020; 182: 59-72.e15. https://doi.org/10.1016/j.cell.2020.05.032 PMID: 32492406

35. Kimhofer T, Lodge S, Whiley L, Gray N, Loo RL, Lawler NG, et al. Integrative Modeling of Quantitative Plasma Lipoprotein, Metabolic, and Amino Acid Data Reveals a Multiorgan Pathological Signature of SARS-CoV-2 Infection. J Proteome Res. 2020 [cited 12 Oct 2020]. https://doi.org/10.1021/acs. jproteome.0c00519 PMID: 32806897

36. Bruzzone C, Bizkarguenaga M, Gil-Redondo R, Diercks T, Arana E, García de Vicuña A, et al. SARSCoV-2 Infection Dysregulates the Metabolomic and Lipidomic Profiles of Serum. iScience. 2020; 23: 101645. https://doi.org/10.1016/j.isci.2020.101645 PMID: 33043283

37. Song J-W, Lam SM, Fan X, Cao W-J, Wang S-Y, Tian H, et al. Omics-Driven Systems Interrogation of Metabolic Dysregulation in COVID-19 Pathogenesis. Cell Metab. 2020; 32: 188-202.e5. https://doi.org/ 10.1016/j.cmet.2020.06.016 PMID: 32610096

38. Schoggins JW, Randall G. Lipids in Innate Antiviral Defense. Cell Host Microbe. 2013; 14: 379-385. https://doi.org/10.1016/j.chom.2013.09.010 PMID: 24139397

39. Abu-Farha M, Thanaraj TA, Qaddoumi MG, Hashem A, Abubaker J, Al-Mulla F. The Role of Lipid Metabolism in COVID-19 Virus Infection and as a Drug Target. Int J Mol Sci. 2020; 21. https://doi.org/ 10.3390/ijms21103544 PMID: 32429572

40. Fan J, Wang H, Ye G, Cao X, Xu X, Tan W, et al. Letter to the Editor: Low-density lipoprotein is a potential predictor of poor prognosis in patients with coronavirus disease 2019. Metabolism. 2020; 107: 154243. https://doi.org/10.1016/j.metabol.2020.154243 PMID: 32320740 
41. Mardinoglu A, Stančáková A, Lotta LA, Kuusisto J, Boren J, Blüher M, et al. Plasma Mannose Levels Are Associated with Incident Type 2 Diabetes and Cardiovascular Disease. Cell Metabolism. 2017; 26: 281-283. https://doi.org/10.1016/j.cmet.2017.07.006 PMID: 28768165

42. Rubino F, Amiel SA, Zimmet $P$, Alberti G, Bornstein S, Eckel RH, et al. New-Onset Diabetes in Covid19. New England Journal of Medicine. 2020; 383: 789-790. https://doi.org/10.1056/NEJMc2018688 PMID: 32530585

43. Bernini $P$, Bertini I, Luchinat $C$, Nincheri $P$, Staderini $S$, Turano $P$. Standard operating procedures for pre-analytical handling of blood and urine for metabolomic studies and biobanks. J Biomol NMR. 2011; 49: 231-243. https://doi.org/10.1007/s10858-011-9489-1 PMID: 21380509

44. Ghini V, Quaglio D, Luchinat C, Turano P. NMR for sample quality assessment in metabolomics. N Biotechnol. 2019; 52: 25-34. https://doi.org/10.1016/j.nbt.2019.04.004 PMID: 31022482

45. PD CEN/TS 16945:2016-Molecular in vitro diagnostic examinations. Specifications for pre-examination processes for metabolomics in urine, venous blood serum and plasma. [cited 12 Oct 2020]. Available: https://shop.bsigroup.com/ProductDetail?pid=000000000030339067

46. Breiman L. Random Forests. Machine Learning. 2001; 45: 5-32. https://doi.org/10.1023/ A:1010933404324

47. Touw WG, Bayjanov JR, Overmars L, Backus L, Boekhorst J, Wels M, et al. Data mining in the Life Sciences with Random Forest: a walk in the park or lost in the jungle? Brief Bioinform. 2013; 14: 315-326. https://doi.org/10.1093/bib/bbs034 PMID: 22786785

48. Verikas A, Gelzinis A, Bacauskiene M. Mining data with random forests: A survey and results of new tests. Pattern Recognition. 2011; 44: 330-349. https://doi.org/10.1016/j.patcog.2010.08.011

49. Wilcoxon F. Individual Comparisons by Ranking Methods. Biometrics Bulletin. 1945; 1: 80-83. https:// doi.org/10.2307/3001968

50. Benjamini Y, Hochberg Y. Controlling the False Discovery Rate: A Practical and Powerful Approach to Multiple Testing. Journal of the Royal Statistical Society Series B (Methodological). 1995; 57: 289-300.

51. Rosenthal R. Parametric measures of effect size. The handbook of research synthesis. New York, NY, US: Russell Sage Foundation; 1994. pp. 231-244.

52. Appropriate statistics for ordinal level data: Should we really be using t-test and Cohen'sd for evaluating group differences on the NSSE and other surveys? | BibSonomy. [cited 12 Oct 2020]. Available: https:// www.bibsonomy.org/bibtex/216a5c27e770147e5796719fc6b68547d/kweiand 